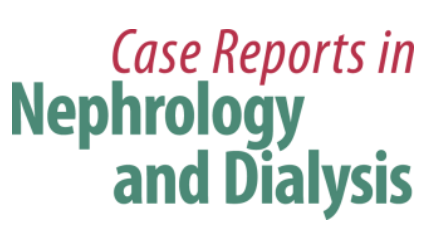

Case Rep Nephrol Dial 2018;8:112-119

DOI: $10.1159 / 000489924$

Published online: June 8, 2018

(C) 2018 The Author(s)

Published by S. Karger AG, Base

www.karger.com/cnd

This article is licensed under the Creative Commons Attribution-NonCommercial 4.0 International License (CC BY-NC) (http://www.karger.com/Services/OpenAccessLicense). Usage and distribution for commercial purposes requires written permission.

\title{
Postembolization Intratumoral Chronic Bleeding, without the Classic CT Feature of Active Extravasation, in Tuberous Sclerosis Complex-Related Renal Angiomyolipoma: Two Case Reports
}

\author{
Xixi Zhang ${ }^{a}$ Ryohei Kuwatsurua, b Hiroshi Toei ${ }^{a}$ Daisuke Yashiro ${ }^{a}$ \\ Shingo Okada ${ }^{\text {b }}$ Hitomi Kato ${ }^{b}$ \\ aDepartment of Radiology, Graduate School of Medicine, Juntendo University, \\ Tokyo, Japan; 'bepartment of Radiology, School of Medicine, Juntendo University, \\ Tokyo, Japan
}

\section{Keywords}

Bleeding $\cdot$ Renal angiomyolipoma $\cdot$ Transcatheter arterial embolization $\cdot$ Tuberous sclerosis complex

\begin{abstract}
Two patients with tuberous sclerosis complex each had multiple bilateral renal angiomyolipomas. After undergoing embolization for a ruptured angiomyolipoma, patient 1 experienced long-lasting abdominal fullness; contrast-enhanced computed tomography (CECT) revealed a large chronic hematoma without contrast extravasation. Patient 2 underwent embolization for the largest right renal angiomyolipoma which contained a chronic hematoma. 2 weeks later, the symptom of abdominal fullness presented, and CECT revealed that the preexisting hematoma had enlarged without contrast extravasation. In both cases, a second embolization of the angiomyolipomas resulted in shrinking of the intratumoral hematomas and alleviation of the associated symptoms. Therefore, chronic postembolization intratumoral bleeding from renal
\end{abstract}




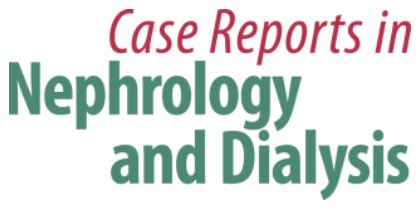

Case Rep Nephrol Dial 2018;8:112-119

DOI: $10.1159 / 000489924$

(c) 2018 The Author(s). Published by S. Karger AG, Basel www.karger.com/cnd

Zhang et al.: Postembolization Intratumoral Chronic Bleeding, without the Classic CT Feature of Active Extravasation, in TSC-Related Renal AML: Two Case Reports

angiomyolipoma may present as a persistently large or growing hematoma with an associated symptom of abdominal fullness but without the typical CECT feature of active extravasation.

(C) 2018 The Author(s)

Published by S. Karger AG, Basel

\section{Introduction}

Renal angiomyolipomas (AMLs) typically comprise disorganized smooth muscle cells, adipose tissue, and abnormal vascular structures [1]. Approximately $95 \%$ of them can be specifically diagnosed based on fat tissue in the tumor detected by computed tomography (CT) or magnetic resonance imaging [2]. Tuberous sclerosis complex (TSC) is an autosomal dominant genetic syndrome with an estimated prevalence ranging from 1 in 6,000 to 1 in 12,000, and about $20 \%$ of patients with renal AMLs have TSC [3]. The blood vessels within AMLs are rigid, tortuous, aneurismal, and prone to rupture [1]. Renal AMLs with pseudoaneurysms larger than $5 \mathrm{~mm}$ are considered to be at high risk of bleeding [4]. Selective transcatheter arterial embolization (TAE) is the first-line treatment option in cases of acute bleeding, with the advantages of preserving normal renal parenchyma and avoiding surgery [5]. Extravasation of contrast agent visible on imaging can confirm the diagnosis and localization of active bleeding [6], enabling selective TAE to be performed in a timely fashion. This report presents two cases with TSC-related renal AMLs in which chronic bleeding after embolization continued without active extravasation.

\section{Case Reports}

Case 1

A 45-year-old woman with TSC had bilateral renal AMLs. She had undergone urgent TAE for a bleeding large left AML at another hospital. Although the ruptured pseudoaneurysm and its parent feeder were selectively occluded by coils, two other arteries feeding the same tumor were not included in the initial procedure. Mammalian target of rapamycin (mTOR) inhibitor was not used after the initial embolization.

Three months after the initial embolization, this patient continued to experience abdominal fullness and was referred to the authors' department. She had stable vital signs, a normal hemoglobin level of $12.3 \mathrm{~g} / \mathrm{dL}$, a normal serum creatinine level of $0.7 \mathrm{mg} / \mathrm{dL}$, and an estimated glomerular filtration rate (eGFR) of $71 \mathrm{~mL} / \mathrm{min} / 1.73 \mathrm{~m}^{2}$. Abdominal nonenhanced CT (NECT) and contrast-enhanced CT (CECT) showed that both kidneys had multiple fat-containing AMLs. The largest AML (11 cm in longest diameter) in the right kidney contained a pseudoaneurysm of $6 \mathrm{~mm}$ in diameter. In the left kidney, the largest AML contained a pseudoaneurysm ( $4 \mathrm{~mm}$ in diameter) and an intratumoral hematoma (volume, $503 \mathrm{~mL}$ ) caused by the previous rupture, with the total size of the target AML and hematoma being $15 \mathrm{~cm}$ in longest diameter. On CECT, the hematoma was seen to be compressing the transverse and descending colon, thus explaining the patient's symptom of abdominal fullness; however, no active extravasation was detected (Fig. 1a). Hematoma volume was calculated with volume analyzer Synapse Vincent software (Fujifilm, Tokyo, Japan). Because neither CECT nor laboratory examinations indicated significant acute bleeding, an elective second TAE was scheduled for both kidneys. 


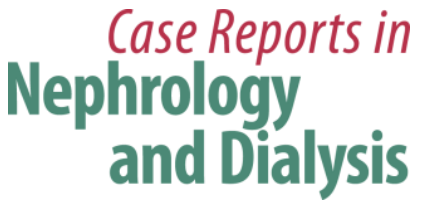

Case Rep Nephrol Dial 2018;8:112-119

DOI: $10.1159 / 000489924$

(c) 2018 The Author(s). Published by S. Karger AG, Basel www.karger.com/cnd

Zhang et al.: Postembolization Intratumoral Chronic Bleeding, without the Classic CT Feature of Active Extravasation, in TSC-Related Renal AML: Two Case Reports

The patient's symptom of abdominal fullness did not diminish over time. The second TAE was performed 6 months after the initial embolization. Selective left renal angiography revealed that the target tumor had two previously untreated feeding arteries and one pseudoaneurysm of $4 \mathrm{~mm}$ in diameter (Fig. 1b). Subsequent superselective angiography for those feeding arteries was performed with the use of a microcatheter (outer diameter, $1.98 \mathrm{Fr}$; Masters Parkway Soft; Asahi Intecc, Nagoya, Japan); no active extravasation of contrast agent was observed. Multiporous gelatin sponge particles ( $1 \mathrm{~mm}$, Gelpart; Nippon Kayaku, Tokyo, Japan) followed by detachable coils (diameter, 1.5-2 mm; length, 3-4 cm; Target Detachable Coils; Stryker Corp., Kalamazoo, MI, USA) were injected to occlude the pseudoaneurysm and feeding arteries of the target AML. After embolization, confirmatory left renal angiography revealed no contrast-enhanced vessels within the target tumor (Fig. 1c). Subsequently, we performed a successful prophylactic embolization of the largest AML in the right kidney during the same procedure.

Within a few days after the second embolization, the patient's symptom of abdominal fullness disappeared. Follow-up CT examinations at 1, 6, and 26 months showed that the chronic hematoma within the left target AML had shrunk (volumes of 280,90 , and $10 \mathrm{~mL}$, respectively), alleviating the compression of the transverse and descending colon. Thirty-one months after the second TAE, the serum creatinine was $0.63 \mathrm{mg} / \mathrm{dL}$ and the eGFR was 78 $\mathrm{mL} / \mathrm{min} / 1.73 \mathrm{~m}^{2}$, indicating that renal function had not worsened.

\section{Case 2}

A 24-year-old woman with TSC had bilateral renal AMLs. About 8 years previously, she had experienced right renal AML bleeding, which resolved with conservative treatment. She came to our department for potential embolotherapy of renal AMLs. She had normal vital signs, a hemoglobin level of $10.0 \mathrm{~g} / \mathrm{dL}$, a normal serum creatinine level of $0.4 \mathrm{mg} / \mathrm{dL}$, and an eGFR of $157 \mathrm{~mL} / \mathrm{min} / 1.73 \mathrm{~m}^{2}$.

Abdominal NECT and CECT examination showed that both kidneys had numerous fatcontaining AMLs with multiple pseudoaneurysms larger than $5 \mathrm{~mm}$. The largest AML in the right kidney was about $23 \mathrm{~cm}$ in longest diameter and contained an intratumoral chronic hematoma (volume, $200 \mathrm{~mL}$ ) without active extravasation (Fig. 2a). Compression of the transverse and ascending colon by the largest AML was detected on CT (Fig. 2a), but the patient did not have associated abdominal symptoms at that time.

Primary TAE was performed for the right kidney because of the chronic intratumoral hematoma. On selective right renal angiography, the multiple AMLs presented as diffuse abnormal tortuous vessels without identifiable tumor margins, and pseudoaneurysms larger than 5 $\mathrm{mm}$ in diameter (the largest one, $7 \mathrm{~mm}$ ) and a predominant portion of tumoral vessels were located at the lower pole (Fig. 2b). Superselective angiography for the lower branches of the renal artery was performed, and the target tumoral vessels and aneurysms were occluded by 1-mm multiporous gelatin sponge particles (Gelpart; Nippon Kayaku) and detachable coils (diameter, 1-3 mm; length, 3-6 cm; Target Detachable Coils; Stryker). Confirmatory right renal angiography showed that most of the abnormal vessels and all of the pseudoaneurysms located in the lower pole had disappeared.

After the initial embolization, mTOR inhibitor therapy was not performed. Two weeks after the initial TAE, the patient complained of abdominal fullness, and laboratory examination showed a decreased hemoglobin level of $7.7 \mathrm{~g} / \mathrm{dL}$. Abdominal NECT and CECT showed that the preexisting intratumoral hematoma in the right kidney had grown (volume, $560 \mathrm{~mL}$ ), leading to increased compression of the transverse and ascending colon (Fig. 2c). Although 


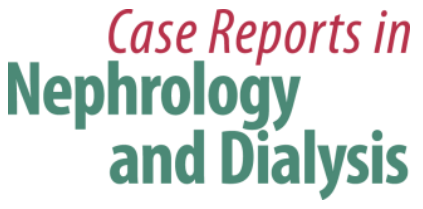

Case Rep Nephrol Dial 2018;8:112-119

DOI: $10.1159 / 000489924$

(c) 2018 The Author(s). Published by S. Karger AG, Basel

www.karger.com/cnd

Zhang et al.: Postembolization Intratumoral Chronic Bleeding, without the Classic CT Feature of Active Extravasation, in TSC-Related Renal AML: Two Case Reports

CECT imaging revealed no active extravasation of contrast agent (Fig. 2c), the enlarged hematoma indicated significant chronic bleeding from the right renal AMLs. Therefore, a second embolization was performed for the right kidney on the same day. On right renal angiography, the absence of active contrast extravasation made the bleeding site undetectable. The tumoral vessels in the middle portion of the kidney, which had the largest pseudoaneurysm (4 $\mathrm{mm}$ in diameter) (Fig. 2d) and the largest feeding artery at that time, were highly suspected as the source of bleeding and were successfully embolized by using the same embolic agents as in the previous TAE (Fig. 2e).

The patient's symptom of abdominal fullness disappeared within a few days after the second embolization. Follow-up CT images at 17 days showed that the right intratumoral hematoma had decreased to $110 \mathrm{~mL}$ and that compression of the transverse and ascending colon had diminished. At 3 months, laboratory examination showed a hemoglobin level of $10.3 \mathrm{~g} / \mathrm{dL}$, the intratumoral hematoma had disappeared on CT, and a prophylactic TAE was performed on the left large AMLs that had pseudoaneurysm(s) larger than $5 \mathrm{~mm}$. Renal function of the patient was normal throughout the postembolization period.

Both patients experienced postembolization syndrome (grade 2; CTCAE ver. 4.03) [7] including fever and pain in the back and flank, but no other complications occurred.

\section{Discussion}

In each of the current cases, after the initial TAE, the renal intratumoral hematoma remained large or grew, and the associated symptom of abdominal fullness was long-lasting or newly emergent; after the second TAE, the hematoma shrank dramatically, leading to quick resolution of the associated symptom and indicating that intratumoral chronic bleeding was present until the second procedure. In each case, because the second TAE was performed on previously untreated feeders, tumoral vessels not included in the initial embolization were causative for the postembolization chronic bleeding.

The typical CT feature of active contrast extravasation within hematomas is an important indicator of active bleeding [8]. However, because the presence of active extravasation is associated with several factors, including pressure difference (blood pressure, potential space), the small size of the disruption in the vessel wall (extent of injury), and the viscosity of the blood (anemia) [6], bleeding from renal AMLs does not always result in active extravasation appearing on CECT. One previous study demonstrated that CECT identified active extravasation in $36 \%$ of ruptured renal AML cases, and angiography identified active extravasation in $44 \%$ of cases [9]. In addition, CT featuring a rapid development of hematoma can suggest the presence of active bleeding [8], and it is easy to identify initial bleeding from renal AMLs by detecting new collections of blood on CT even without active extravasation. Nevertheless, when chronic bleeding from a renal AML occurs in the context of a preexisting hematoma and presents no active extravasation on CECT, it may be hidden by the previous hematoma and need more careful monitoring. In the two current cases, the only sign of chronic bleeding from postembolization renal AML was a persistently large or growing hematoma on CECT imaging, with no active extravasation of contrast agents.

Because the kidneys are located in the retroperitoneal space, it is uncommon for a renal hematoma to cause intestinal symptoms. In two previously reported cases, serious intestinal complications (duodenal obstruction) were caused by renal hematoma due to a bleeding renal AML or severe blunt renal trauma and were ameliorated after image-guided drainage of the hematoma $[10,11]$. In the two cases reported here, the renal chronic hematomas were each 


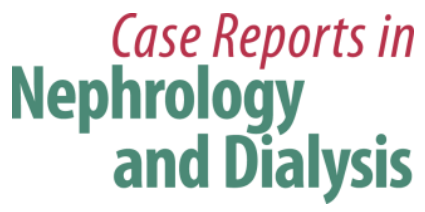

\begin{tabular}{l|l}
\hline Case Rep Nephrol Dial 2018;8:112-119 \\
\hline DOI: 10.1159/000489924 & $\begin{array}{l}\text { @ 2018 The Author(s). Published by S. Karger AG, Basel } \\
\text { www.karger.com/cnd }\end{array}$ \\
\hline
\end{tabular}

Zhang et al.: Postembolization Intratumoral Chronic Bleeding, without the Classic CT Feature of Active Extravasation, in TSC-Related Renal AML: Two Case Reports

located within a large AML that allowed compression of the intestines; the persistent large hematoma (in case 1) or the growing hematoma (in case 2) compressed the intestines and caused the patients' long-lasting or newly emergent symptoms of abdominal fullness.

Renal AMLs associated with TSC are usually multiple and bilateral, and in these cases, preservation of the renal parenchyma is essential during the treatment of bleeding from AML. As a safe and minimally invasive procedure, superselective TAE of renal AML can preserve normal renal parenchyma as much as possible and can be performed multiple times $[5,12]$. In fact, in each case reported here, despite multiple TAEs, chronic bleeding from renal AML was treated with consequent amelioration of the associated patient symptom, contralateral renal AML(s) with pseudoaneurysm(s) $>5 \mathrm{~mm}$ were prophylactically embolized, and there was no significant deterioration of renal function in the postembolization period and no other complication except postembolization syndrome.

\section{Conclusion}

For cases with TSC-related renal AMLs that have tumoral vessels untreated by previous embolization, when considering reasons for the symptom of abdominal fullness, the possibility of postembolization chronic intratumoral bleeding must be taken into account, and it may present as a persistently large or growing hematoma but without the typical CECT feature of active extravasation.

\section{Statement of Ethics}

This report was approved by the institutional review board (JHS 17-0029) and the requirement for informed consent was waived.

\section{Disclosure Statement}

The authors declare no conflict of interest.

\section{References}

1 Lendvay TS, Marshall FF. The tuberous sclerosis complex and its highly variable manifestations. J Urol. 2003 May;169(5):1635-42.

2 Krishnan A, Kaza RK, Vummidi DR. Cross-sectional Imaging Review of Tuberous Sclerosis. Radiol Clin North Am. 2016 May;54(3):423-40.

3 Umeoka S, Koyama T, Miki Y, Akai M, Tsutsui K, Togashi K. Pictorial review of tuberous sclerosis in various organs. Radiographics. 2008 Nov-Dec;28(7):e32.

4 Yamakado K, Tanaka N, Nakagawa T, Kobayashi S, Yanagawa M, Takeda K. Renal angiomyolipoma: relationships between tumor size, aneurysm formation, and rupture. Radiology. 2002 Oct;225(1):78-82.

5 Chatziioannou A, Gargas D, Malagari K, Kornezos I, Ioannidis I, Primetis E, et al. Transcatheter arterial embolization as therapy of renal angiomyolipomas: the evolution in 15 years of experience. Eur J Radiol. 2012 Sep;81(9):2308-12.

6 Hamilton JD, Kumaravel M, Censullo ML, Cohen AM, Kievlan DS, West OC. Multidetector CT evaluation of active extravasation in blunt abdominal and pelvic trauma patients. Radiographics. 2008 0ct;28(6):1603-16.

7 US Department of Health and Human Services. National Institutes of Health, National Cancer Institute: Common Terminology Criteria for Adverse Events (CTACE) Version 4.03. 2010. Available from: 
https://evs.nci.nih.gov/ftp1/CTCAE/CTCAE_4.03_2010-06-14_QuickReference_8.5x11.pdf. Accessed: June 6, 2018.

8 Park HJ, Shin JH, Han KC, Yoon HK, Ko GY, Sung KB. Transcatheter Arterial Embolization of Angiographically Visible and Occult Renal Capsular Artery Hemorrhage in 28 Patients. J Vasc Interv Radiol. 2016 Jul;27(7):973-80.

9 Duan XH, Zhang MF, Ren JZ, Han XW, Chen PF, Zhang K, et al. Urgent transcatheter arterial embolization for the treatment of ruptured renal angiomyolipoma with spontaneous hemorrhage. Acta Radiol. 2016 Nov;57(11):1360-5.

10 Park JK, Yang SO, Park SC. Duodenal obstruction by retroperitoneal hematoma induced by severe blunt renal trauma. J Urol. 2001 Jun;165(6 Pt 1):1993-4.

11 Teoh JY, Chan NH, Cheung HY, Hou SS, Ng CF. Bleeding renal angiomyolipoma presenting as duodenal obstruction. Int Urol Nephrol. 2013 Aug;45(4):975-7.

12 Halpenny D, Snow A, McNeill G, Torreggiani WC. The radiological diagnosis and treatment of renal angiomyolipoma-current status. Clin Radiol. 2010 Feb;65(2):99-108. 


\section{Case Reports in Nephrology and Dialysis}

Case Rep Nephrol Dial 2018;8:112-119

DOI: $10.1159 / 000489924$

(c) 2018 The Author(s). Published by S. Karger AG, Basel www.karger.com/cnd

Zhang et al: Postembolization Intratumoral Chronic Bleeding, without the Classic CT Feature of Active Extravasation, in TSC-Related Renal AML: Two Case Reports
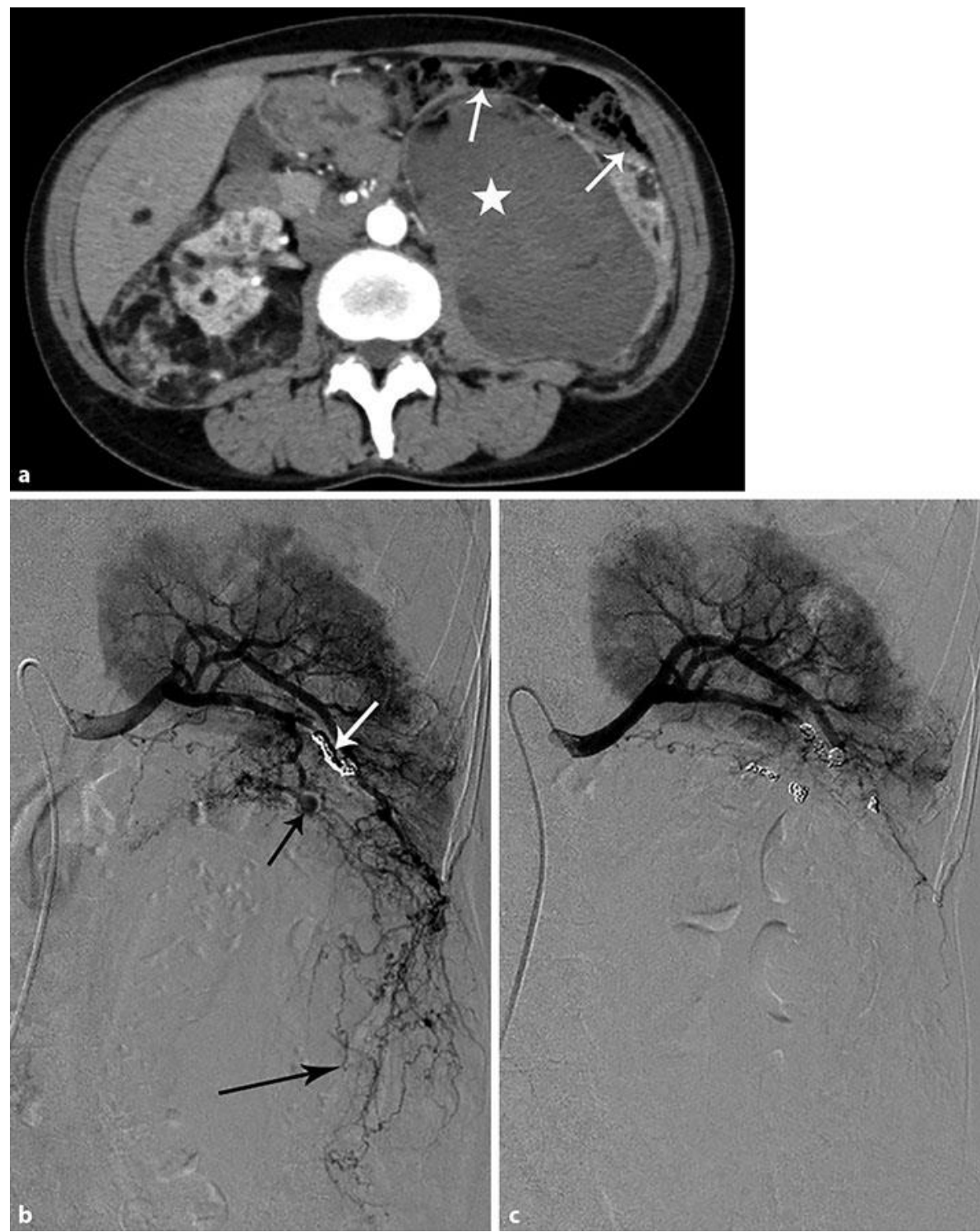

Fig. 1. a Axial CECT image obtained 3 months after emergency embolization of left renal AML. A large chronic intratumoral hematoma (star) without contrast extravasation is compressing the transverse and descending colon (arrows). b Left renal angiography before the second TAE shows abnormal tortuous vessels (long black arrow) and a pseudoaneurysm (short black arrow) which were not treated in the initial procedure. Coils (white arrow) used for the ruptured pseudoaneurysm and its parent artery during the initial TAE can also be seen. c Confirmatory left renal angiography after the second TAE. All abnormal vessels and the pseudoaneurysm of the target tumor have disappeared. 


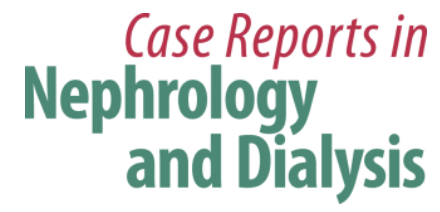

Case Rep Nephrol Dial 2018;8:112-119

DOI: $10.1159 / 000489924$

(c) 2018 The Author(s). Published by S. Karger AG, Basel www.karger.com/cnd

Zhang et al: Postembolization Intratumoral Chronic Bleeding, without the Classic CT Feature of Active Extravasation, in TSC-Related Renal AML: Two Case Reports
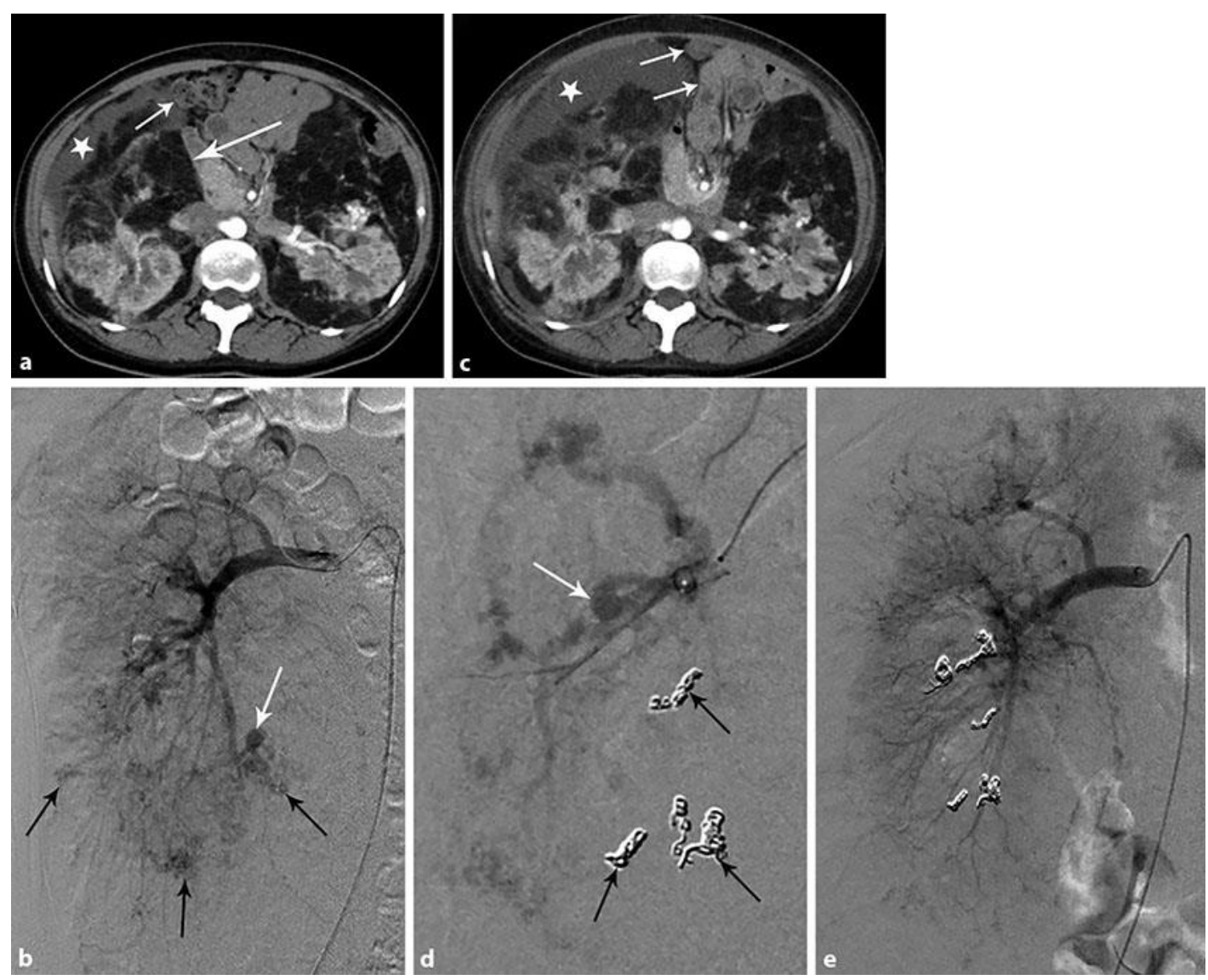

Fig. 2. a Axial CECT image obtained before the initial TAE. The largest AML (long white arrow) in the right kidney with an intratumoral chronic hematoma (star) is compressing the transverse and ascending colon (short white arrow). b Right renal angiography before the initial TAE. Right renal AMLs present as diffuse tortuous vessels without identifiable tumor margins. A predominant portion of abnormal vessels (black arrows) and the largest pseudoaneurysm (white arrow) are located at the lower pole. c Axial CECT image obtained 2 weeks after the initial TAE. The chronic hematoma (star) has enlarged, increasing the compression of the transverse and ascending colon (arrows). No contrast extravasation within the hematoma can be seen. $\mathbf{d}$ Superselective angiography of one major feeder located in the middle portion of the kidney, performed before the second TAE. It shows the largest pseudoaneurysm ( $4 \mathrm{~mm}$ in diameter, white arrow), from which there is no extravasation of contrast agent. Coils (black arrows) used during initial TAE for pseudoaneurysms and tumoral vessels located in the lower pole can be seen. e Confirmatory right renal angiography after the second TAE. Most tumoral vessels and all aneurysms located in the middle and lower portions of the right kidney have disappeared. 